\title{
Practical Model Checking of a Home Area Network System: Case Study
}

\author{
Soufiane Zahid ${ }^{1}$, Abdeslam En-Nouaary ${ }^{1}$ and Slimane Bah ${ }^{2}$ \\ ${ }^{1}$ Institut National des Postes et Télécommunication, Rabat, Morocco \\ ${ }^{2}$ Ecole Mohammadia d'Ingénieurs, University Mohammed V, Rabat, Morocco
}

\begin{abstract}
The integrated communication infrastructure is the core of the Smart Grid architecture. Its two-way communication and information flow provides this network with all needed resources in order to control and manage all connected components from the utility to the customer side. This latter, named the Home Area Network or HAN, is a dedicated network connecting smart devices inside the customer home, and using different solutions. In order to avoid problems and anomalies along the process life cycle of developing a new solution for HAN network, the modeling and validation is one of the most powerful tools to achieve this goal. This paper presents a practical case study of such validation. It intends to validate a HAN SDL model, described in a previous work, using model checking techniques. It introduces a method to translate the SDL model to a Promela model using an intermediate format IF. After the generation of the Promela model, verification is performed to ensure that some functional properties are satisfied. The desired properties are defined in Linear Temporal Logic (LTL), and DTSPIN (an extension of SPIN with discrete time) model checker is used to verify the correctness of the model.
\end{abstract}

ACM CCS (2012) Classification: Software and its engineering $\rightarrow$ Software organization and properties $\rightarrow$ Software functional properties $\rightarrow$ Formal methods $\rightarrow$ Model checking

Networks $\rightarrow$ Network types $\rightarrow$ Home networks

Keywords: Smart Grid, Promela generation, Formal modeling, V\&V, SPIN, LTL

\section{Introduction}

Smart Grid is an intelligent power network based on information and communication technologies in order to monitor, optimize and control all functional units from electricity generation to end-customers. Many international organizations and government institutions around the world have been encouraging the use of Smart Grids, and proposed their own models and roadmaps for this. In particular, the end-user side, or Home Area Network (HAN), represents a challenge for these organizations in order to help customers to reduce their energy consumption and cost, and to maximize the transparency and reliability of the energy supply chain.

In a previous work [1], a communication infrastructure model for the Smart Grid was presented. This model is based on international guidelines proposed by IEEE, ITU and NIST. It focuses on the communication aspect of Smart Grid and its services as defined by the U.S. Department of Energy (DOE) [2]. This previous work aimed to analyze, study and understand the communication relationship between the system components. It explained the functioning of essential units and the interaction between them, and it proposed a set of possible technologies and standards for each link between the entities. The analysis was divided into three parts depending on the network studied: Home Area Network (HAN), Neighborhood Area Network (NAN) or Wide Area Network (WAN). 
The HAN network is one of the most important parts in Smart Grid. Many researches around the world are focusing on this network and they are trying to investigate its potential benefits for both customers and providers. So, the analysis of the previous architecture was started by modeling the HAN network [3] using SDL (Specification and Description Language). Then, the resulting model was verified and validated by the reachability analysis technique, with the support of IBM Rational SDL suite, to detect dead blocks and to ensure that all the branches are reachable. The bit-state exploration was used to find unexplored branches in the model. If they were found, the model was corrected and the verification was rerun until a model free of dead blocks was obtained. However, at this stage, the properties of the system have not been verified yet. In fact, the task of validating implementation of the model is generally much harder. This paper focuses only on some properties in relation with the Demand Response service (which will be described in the next section). These properties will be detailed later. Unfortunately, the SDL language does not allow such validation [4]. This work aims to perform the model checking [5] step using an extension of one of the most powerful tools in this field, which is SPIN model checker. It will be used to verify the desirable temporal properties of the model, expressed as LTL formulas, to evaluate the system requirements. The SDL model must be translated into Promela (the input language for SPIN), however, this translation is not as trivial as it may seem. There are some fundamental differences between these two languages. In fact, SDL supports hierarchical structure, whereas Promela is a flat language, with one level of hierarchy. Also, some SDL data types and constructs cannot be represented trivially in Promela, because they usually include additional information in relation with the system hierarchy.

This case study introduces one approach to generate the Promela model from the SDL one, using an intermediate format IF. The reason behind this choice is that the transformation from SDL to IF is intuitive and simple compared to the translation from SDL to Promela directly. Also, it provides a common model between various languages adopting different description styles (e.g. SDL, UML). After the translation of the SDL model into Promela, verifi- cation is done to ensure that some functional properties, represented as LTL properties, are satisfied. This approach can be applied to any model developed in the SDL language.

The remainder of this paper is organized as follows. The next section presents the background of the work. It gives a brief description of the HAN architecture and its SDL modeling, then, it introduces some works related to Promela generation from SDL. Section 3 details the translation approach via two big steps. The first step highlights transformation of the SDL model into the intermediate format IF. It explains some translation features concerning the structure, the behavior and the data. The second one introduces the if $2 \mathrm{pml}$ tool used to generate the Promela model. Section 4 presents the model checking of the generated model. It describes some LTL properties to be checked and the result of this verification. The last section concludes the paper and presents the future works.

\section{Background}

The Home Area Network or HAN is the customer side in a Smart Grid network. It is contained within the user's home and connects all appliances and electrical vehicles to a common network. It contains other solutions, such as software applications to manage and control these devices, as well as renewable energy resources and energy storage equipment.

A previous work [1] presented HAN architecture and its mandatory and essential components. It introduced the possible and well-used communication technologies and standards for each section. This architecture brings a lot of benefits to the customers. They can take advantage of the services provided by utilities. The most important one is the service DR (Demand Response). It is responsible for reducing the peak loads, when the network is under stress, by minimizing the energy consumption in response to an increase in the price of electricity [6]. So, each customer may participate in one or more DR programs. The raisons behind encouraging customers to participate in such programs are different. They help them reduce the bills price, avoid blackouts and increase their sense of responsibility. DR programs are classified into two major categories, namely Incentive-based 


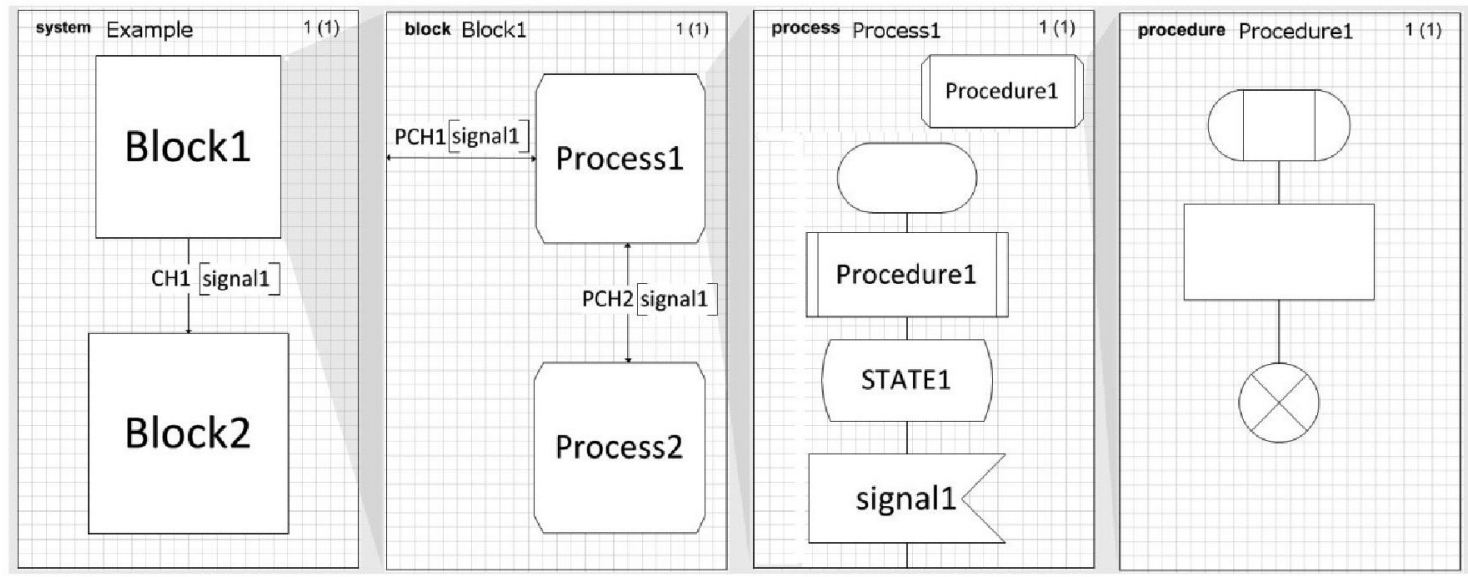

Figure 1. A structural vision of an SDL model [10].

Programs and Time-based Programs; each category contains several programs [7].

This architecture was modeled using the SDL language [8]. The latter is a standard language for specification and description of reactive and distributed systems. A model described in SDL is composed of one or several blocks. Each block contains either other blocks or processes. The behavior of the processes is detailed using Extended Finite State Machine (EFSM) [9]. Unlike FSM that always performs a transition on a given input from one state to another, an EFSM however only performs a transition when a given set of conditions have been satisfied. It means that the transition can be expressed by a set of trigger conditions (e.g. if statements). If trigger conditions are all satisfied, the transition is fired, bringing the machine from the current state to the next state and performing the specified data operations. On the other hand, the procedure behavior is described by FSM. It is similar to the one known from programming languages. It is created when a procedure call is interpreted, and it dies when it terminates. Signals in the system are exchanged between blocks via channels, and between processes via signal routes. Figure 1 resumes this architecture [10].

The SDL model of the HAN architecture, cited in a previous contribution [3], consists of six blocks as shown in Figure 2. Each entity represents one of the HAN's components, namely:

- Appliance: refers to all devices inside the home,

- PEV: or Plug-in Electrical Vehicle,
- IHD: or In-Home Display which is an interface to control the appliances and show statistics,

- HEMS: or Home Energy Management System, it stores data about all the connected appliances and PEV, and manages the DR signals from the utility,

- SM: or Smart Meter, it displays the energy consumption of the customer,

- ESI: or Energy Services Interface, which plays the role of the gateway between the home and the utility network.

The SDL system interacts with system environment via 5 channels. Channels $\mathrm{C} 1, \mathrm{C} 4$ and C9 represent the customer interaction with the HAN; channel C11 is related to the energy generated by renewable sources; and the last one is C14 which connects the HAN with the NAN. This model was verified using the reachability analysis techniques and the verification against a given MSC (Message Sequence Charts) in order to detect dead blocks and verify specific scenarios, respectively [3]. However, no verification of the system behavior has been made yet. In fact, the SDL language does not allow such verification [4]. Therefore, this paper intends to perform model checking [11] to verify the model behavior. For this purpose, one of the existing model checkers must be chosen.

Model checkers are the tools that help in this type of formal verification. In literature, there is a very large number of these tools. Authors in [12] compared six model checkers, namely: Alloy [13], CADP [14], FDR2 [15], NuSMV [16], 
ProB [17], SPIN [18]. They specified a single case study using each of those tools and evaluated the characteristics of the system in order to identify the most suitable model checker for the information systems. However, the checked properties represented only specific information system properties. Another work [19] verified an algorithm for Automatic Train Supervision using ten model checkers, namely: UMC [20], SPIN, NuSMV, mCRL2 [21], CPN Tools [22], FDR4 [23], CADP, TLA+ [24], UPPAAL [25] and ProB. It highlighted the commonalities and differences among the modeling languages considered, and presented the impact of these languages on the model.

Therefore, the choice between the different types of model checkers is not an easy task. It depends on the system architecture, the properties to be verified and other system characteristics. Generally, each modeling language can be translated (manually and/or automatically) into any verification language. However, only smaller pairs are in use [26]. The SDL/Promela is one of those pairs [26]. Since Promela is the input verification language of SPIN [27], this model checker is chosen in the verification step. The other model checkers could be used in this case study, but they are not the best choice. For example, the transformation from SDL to intermediate language $\mathrm{SDL}_{\mathrm{xta}}$ and then to "xta" language used in UPPAAL [25] tool is a very complex process [28]. Also, in the SMV language (used in NuSMV), all assignments, parameters or array indexes should be constant. So, specifications may be longer than in Promela [12], because each case has to be explicitly written. The range verification time in SPIN compared to the ten other model checkers is reasonable [19]. This parameter represents the time needed to terminate the verification. It is expressed as a range because it actually depends on the specific design approach adopted, on the specific formulas being evaluated, and on the specific options used during the tool execution. On the other hand, and unlike many model checkers, SPIN does not perform the verification itself,

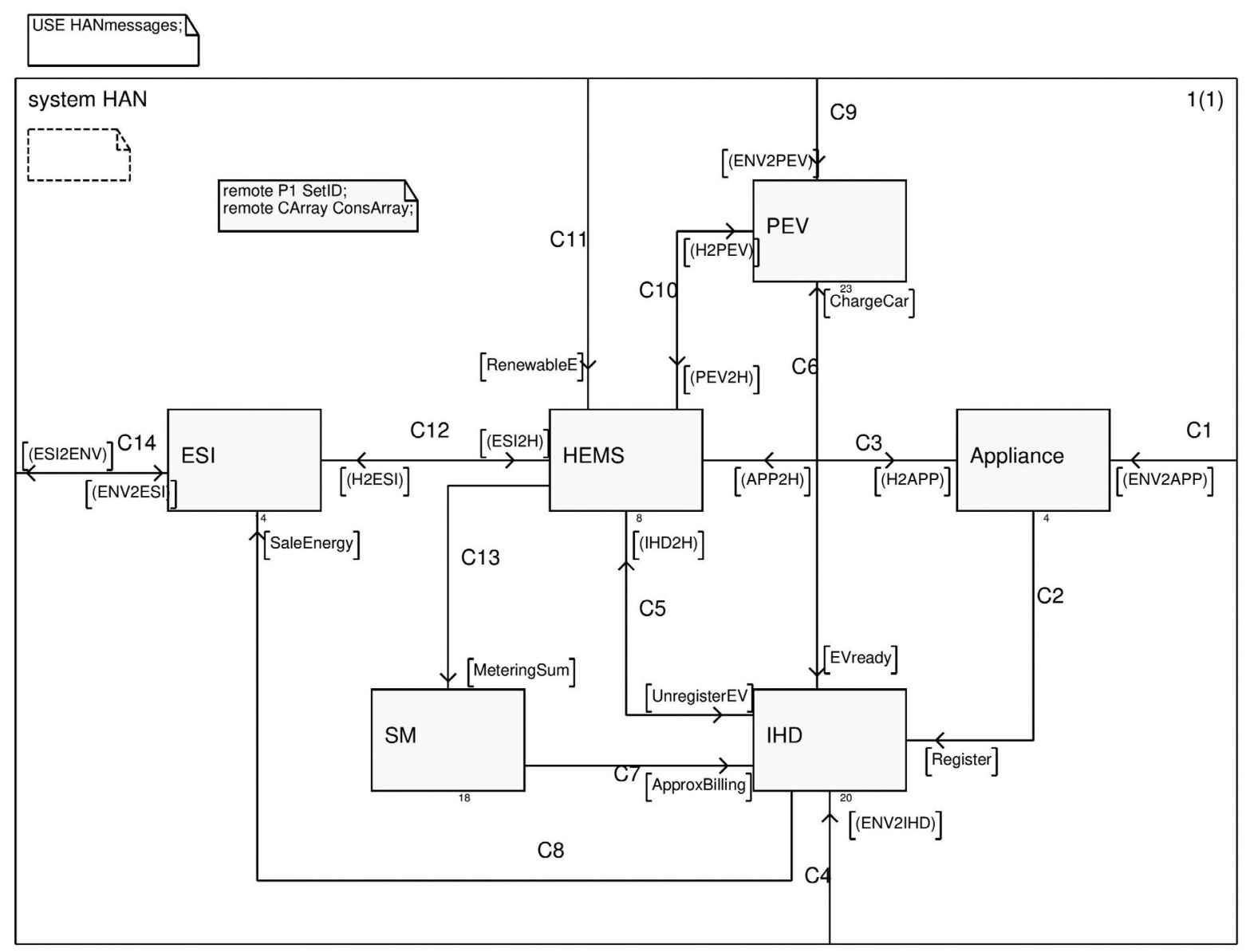

Figure 2. The system view of the HAN SDL model. 
but it generates $\mathrm{C}$ sources for the problem. This technique allows improving performance and saving memory [18]. SPIN also offers a large number of options to further speed up the model checking process.

In order to use SPIN, the SDL model needs to be translated into Promela. This transformation is not as trivial as it seems. Many methods found in literature have addressed this problematic. The most popular method is the use of an intermediate format IF [29]. Firstly, the model is translated into IF using sdl2if tool, then the Promela model is generated from the resulting model using if2pml tool. This technique lacks support of some important SDL features like the "save" operator and the Timer. To solve the "save" operator problem, the authors in [30] proposed an extension of if2pml to translate this operator from IF to Promela. The extension uses additional local queues to which the saved signals are sent. Also, the sdl2if tool works only with ObjectGeode (obsolete product) API and requires license file. It is not available for IBM Rational SDL Suite, which is the successor of ObjectGeode.

Another interesting contribution was introduced in [31]. The authors presented their project to directly generate Promela model from the SDL specification. This work resulted in the implementation of automated generation tool named sdl2pml [32]. The latter is, to the best of the authors' knowledge, the only tool that supports the translation of all SDL constructs. Unfortunately, this tool is not yet available as free/commercial product.

In this paper, the adopted approach is based on the intermediate format IF. The motivation behind this choice is to provide a common model between various languages adopting different description styles (e.g. SDL, UML). The model will be translated manually into IF language and then the if $2 \mathrm{pml}$ tool will be used to generate Promela script.

\section{Generation of the Promela Model}

Generally, after creating an SDL model of a system and validating it via reachability analysis techniques, a designer is interested in the verification of specific proprieties. One of the well-known methods is the model checking. This paper aims to check exhaustively and automatically whether the HAN model meets given specifications. However, modifications must be made to this model to be compatible, after transformation, with SPIN. In fact, SPIN requires a complete system, with no interactions with the environment. This section describes the required modifications in order to create a closed model from the model described above and the transformation process.

\subsection{A Closed Model for HAN}

In order to achieve a closed architecture, the specification is supplemented with a model of its environment as shown in Figure 3. This block contains three processes which represent the interaction of customer, utility and renewable energy sources with the system. Figure 4 represents the finite state machine of one of these processes.

The environment block defines a set of signals to execute all possible paths which can be checked during the verification step. The standard SDL key word "ANY" is used to create all the decisions in this process. In fact, in order to run the model checking process in an automated way, the user intervention must be eliminated. The model must not contain any decision statements that need external intervention. All decisions are nondeterministic in this block. Also, the "environment" is not limited by the order in which the signals will be sent.

On the other hand, all decisions described by informal text were replaced by nondeterministic decision statements. The reserved word "ANY", again, is used to transform a simple decision to a nondeterministic decision. The rest of the model remains unchanged.

This new model was also validated using the three techniques described in [3]. All errors were detected and corrected. It should be noted that this is only an example of one possible model for the block "environment". And if the "environment" block becomes more complex, the verification becomes complicated too. 


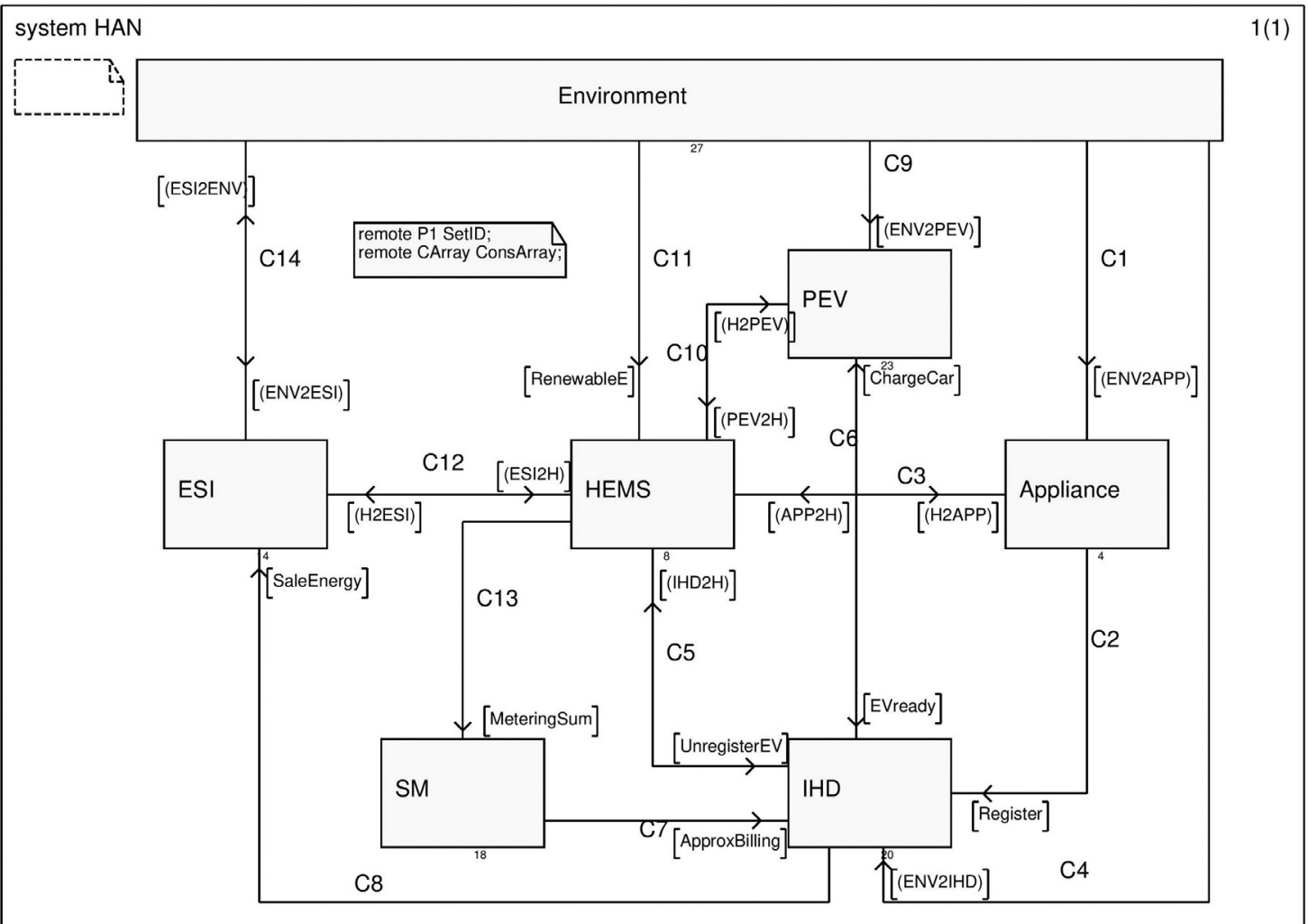

Figure 3. SDL model of HAN and its environment.

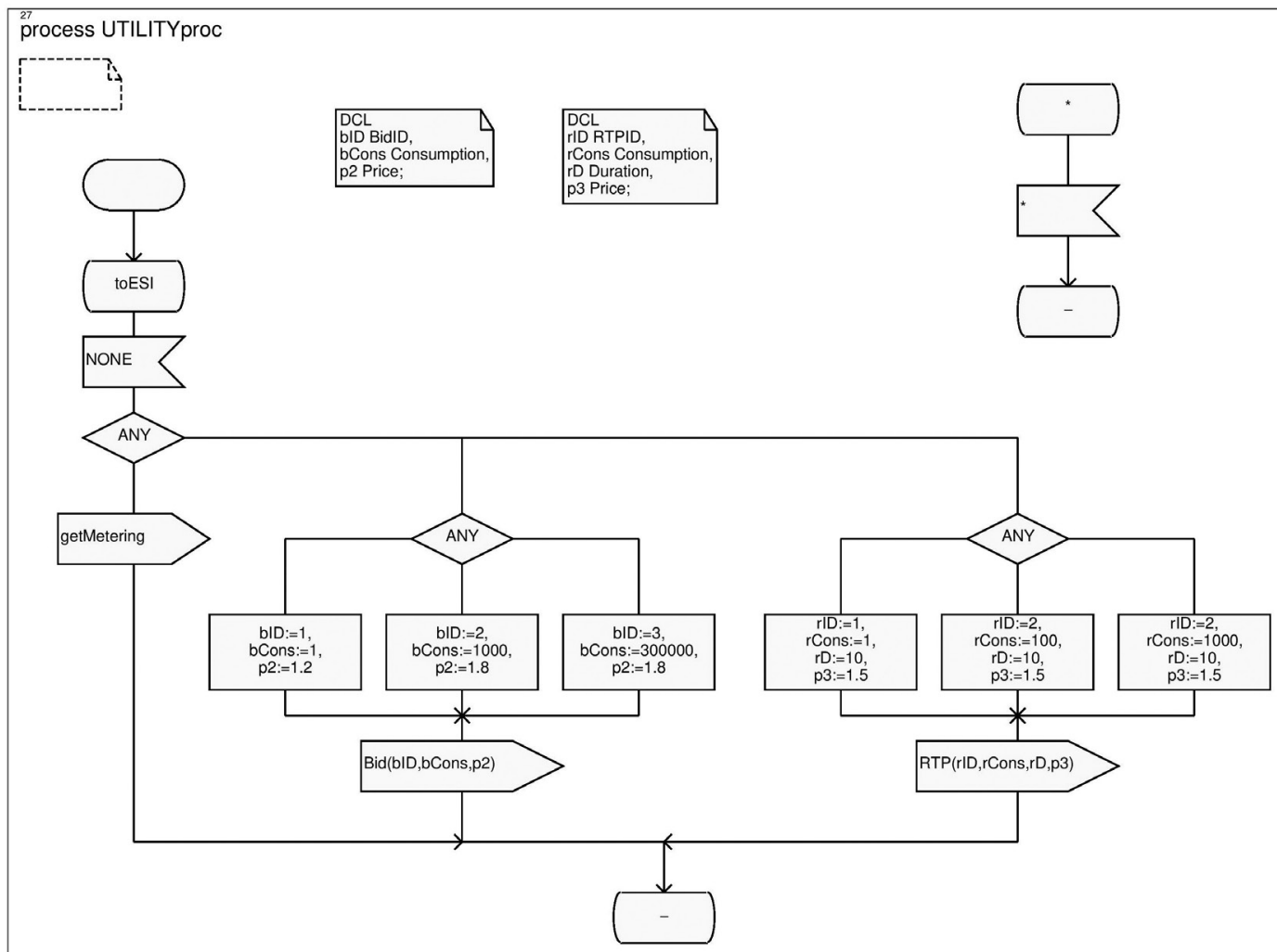




\subsection{Promela Model}

As mentioned before, the model is firstly translated into an intermediate format IF. This subsection explains the translation of some SDL features concerning the structure, the behavior and the data type.

IF was developed to sit between high-level specification languages, such as SDL and UML, and tool specific internal representations [29]. Thus, IF representations can be passed between the tools, and translated into other languages, for example, SDL specifications can be analyzed using SPIN as described in this paper. Another example is the UML, where the mapping is done in a way that all runtime entities (objects, call stacks, pending messages, etc.) are identifiable as a part of the IF model's state [33]. Moreover, translating high-level languages into IF may also allow extending the model with other features missed in the original language.

The structures of SDL and IF are different. In fact, an SDL model may contain blocks, processes and services. Elseways, the IF models are flat and they are composed only of processes at one level. However, at the execution time, even an SDL model is composed of processes that react with each other. Thus, each SDL process is translated into an equivalent IF process. The remote exported/imported variables defined inside processes are declared only once at the IF system level. Thus, all other processes of the system can use them. Since the IF does not support the dynamic creation of process, only one instance of these processes is created. The dynamic creation will be added later, using the "run" operator in Promela.

In IF, there are two types of states: "stable" and "nostable". By default, all the SDL states are translated into "stable" states. The "nostable" type is used to model supplementary states added in order to divide a long transition into small transitions. In these states, the process blocks the others and continues to execute its code until it reaches a stable state. Figure 5 shows an example of this decomposition. The transition from the state waitOK to the state ready is long. So, two "nostable" states, namely, q1_3 and q1_4, were added. Now, in each sub-transition there is only a simple set of actions (receive signal, condition test, send signal). Generally, "nostable" states are added before every condition test.

An IF transition represents the minimal path between two IF states (either stable or nostable), and contains all triggers and actions defined on that path in the same order. It has higher priority than time progress; so, by default, its type is "eager". The simple input signals are directly represented with an asynchronous IF input. For other types (e.g. spontaneous, continuous, priority), other auxiliary parameters are needed, such as PID, to translate them. For instance, the NONE signal is translated by an assigning the PID of the current process to the sender. No input part is generated in this case i.e. the input part of the corresponding IF transition is empty.

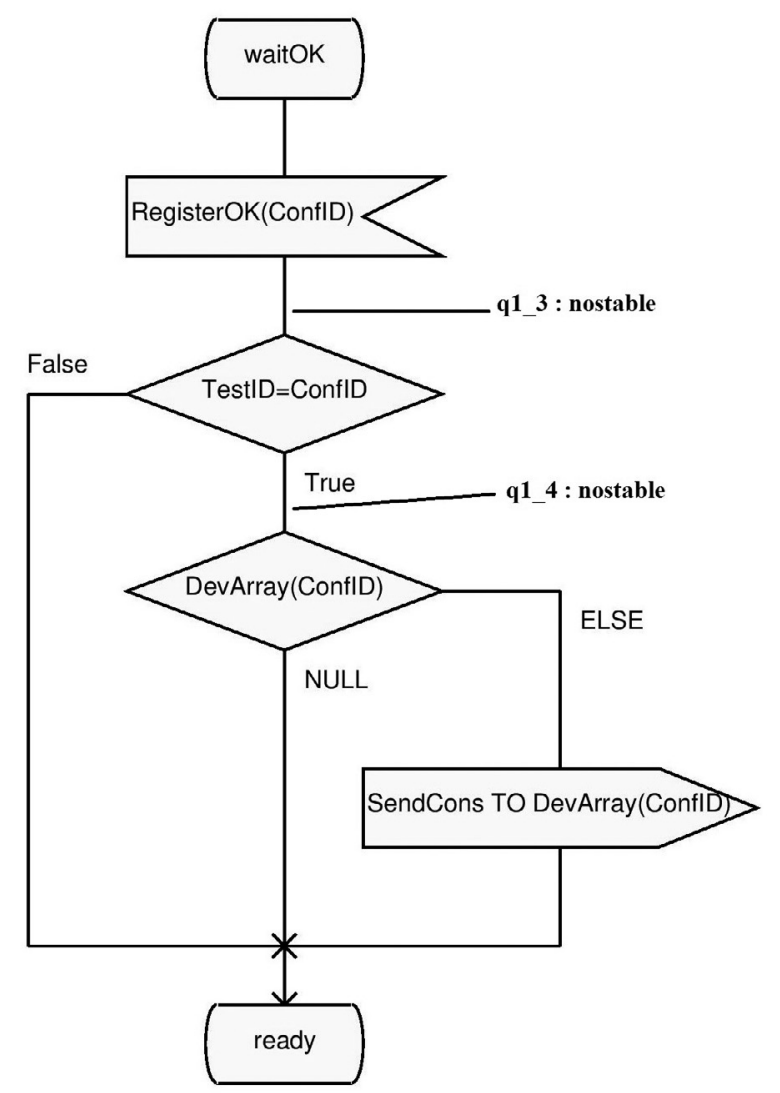

Figure 5. Nostable state example.

The SDL channels are translated using IF buffers. Each process has a unique input buffer through which messages are received. A buffer is defined by name, the serving discipline (i.e. queue, stack, set, and bag) and the set of signals that can be transported. The buffers are global and are used to transport signals between the 
processes. Thus, each process can specify to which buffer a signal should be written or from which buffer a signal should be read.

On the other hand, each predefined data type used in SDL model has its equivalent in IF. The abstract data types are also translated into an IF ADT with the same signature. However, if the latter contains only predefined or implicit operators, it is translated into an enumerated type. Table 1 gives an example of the translation features. For the translation of channel C3, only the definition of one buffer is given. Normally, the equivalent representation contains five buffers, because there are two processes in the block Appliance, and three processes in the block HEMS. However, for the sake of space and simplicity, only one buffer is represented.

The second step is the generation of Promela model. Promela is a verification modeling language introduced by Gerard J. Holzmann [34]. The behavior of a system described in Promela is presented inside the processes. There is no notion of block, thus there is only one level, i.e. the process level. This language allows the dynamic creation of concurrent processes to mod- el, for example, distributed systems. Data are exchanged between processes through message channels. They can be defined to be synchronous (i.e., rendezvous), or asynchronous (i.e., buffered). Promela models can be analyzed with the SPIN model checker, to verify that the modeled system produces the desired behavior, as shown in the rest of this paper.

Generation of the Promela model from IF model is achieved with the if2pml tool. Thus, the Promela model is generated automatically from the IF script created earlier. However, as mentioned before, one change is made to this model. The dynamic creation of processes, using the "run" operator, is added because IF does not support it. However, the if $2 \mathrm{pml}$ has made another change. In fact, Promela lacks the predefined type "real". So, the tool changes all the real variables into natural variables. This modification does not influence the model behavior. Indeed, the variables used (e.g. price, charge) could be either real or natural.

Another aspect missed in Promela is the save operator. This problem has been investigated by researchers in [4]. They implemented an exten-

Table 1. SDL to IF translation features example

\begin{tabular}{|c|c|}
\hline SDL representation & IF representation \\
\hline $\begin{array}{l}\text { NEWTYPE AppType } \\
\text { LITERALS CTRL, CRITICAL, NULL } \\
\text { ENDNEWTYPE; }\end{array}$ & AppType = enum CTRL, CRITICAL, NULL; \\
\hline $\begin{array}{l}\text { SYNTYPE } \\
\text { DevPriority = Integer CONSTANTS 0:2 } \\
\text { ENDSYNTYPE; }\end{array}$ & DevPriority $=$ range $0 . .2$ \\
\hline $\begin{array}{l}\text { NEWTYPE } \\
\text { AppArray ARRAY(AppID, PID) } \\
\text { ENDNEWTYPE; }\end{array}$ & AppArray $=\operatorname{array}[0 . .100]$ of pid; \\
\hline $\begin{array}{l}\text { SIGNAL } \\
\text { PowerOFF(AppID); }\end{array}$ & $\begin{array}{l}\text { SIGNAL } \\
\text { PowerOFF(pid); }\end{array}$ \\
\hline $\begin{array}{l}\text { channel C3 } \\
\text { from Appliance } \\
\text { to HEMS } \\
\text { with } \\
\text { Unregister, DeleteDev, MeteringData; } \\
\text { from HEMS } \\
\text { to Appliance } \\
\text { with } \\
\text { RegisterOK, Shutdown; } \\
\text { Endchannel C3; }\end{array}$ & 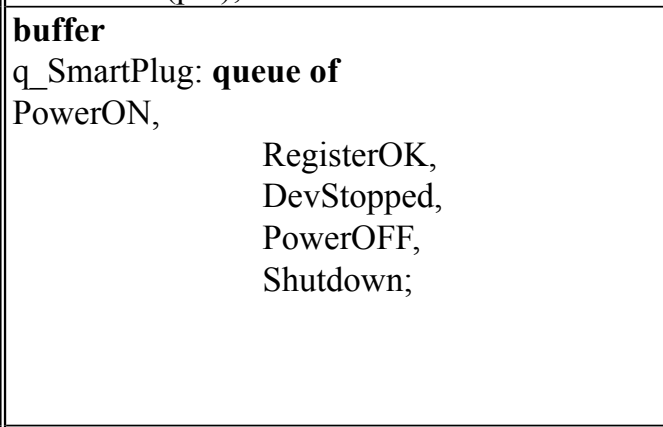 \\
\hline $\begin{array}{l}\text { state DecisionState; } \\
\text { input NONE; }\end{array}$ & $\begin{array}{l}\text { From DecisionState : eager } \\
\text { do sender := DemandResponse }\end{array}$ \\
\hline
\end{tabular}


sion of if $2 \mathrm{pml}$ that uses additional local queues to which the saved signals are sent. In this work, this algorithm is not implemented, however, the XSpin (graphical interface for SPIN) options are used to simulate the save operator during the verification step. The "Queue" options allow either saving the new, not used messages or discarding them. So, in this case study, the choice of saving them is adopted to simulate the SDL save operator.

While Promela has no notion of time, the new version DTPromela [27] is used in this case study to describe real-time proprieties of the HAN model. In DTPromela, a new data type called timer is introduced. It is used to declare the variables that represent discrete-time countdown timers, and then to model quantitative aspects of SDL timers. By default, the if $2 \mathrm{pml}$ tool translates SDL models into this language. The new definition of a timer is added to the system as a Promela macro, contained within a header file. The DTPromela model is then verified using the SPIN extension: DTSPIN.

To illustrate the transformation from SDL to Promela (or DTPromela), Table 2 gives an example using the process "AMIint". An IF process with the same name as the original process was defined. It was associated with a default input queue "q_AMIint". Because the "Sender" variable is missed in IF, additional variable of type PID was defined to represent it explicitly.

Table 2. SDL to Promela translation example.

\begin{tabular}{l}
\hline SDL representation: \\
\hline \hline PROCESS AMIint $(1,1) ;$ \\
DCL \\
$\quad$ SumCons Consumption, \\
$\quad$ HANCons Consumption := $;$ \\
START; \\
NEXTSTATE ready; \\
STATE ready; \\
INPUT MeteringSum(SumCons); \\
TASK HANCons := SumCons; \\
NEXTSTATE -; \\
INPUT getMetering; \\
OUTPUT SendMetering(HANCons); \\
NEXTSTATE -; \\
ENDSTATE; \\
ENDPROCESS AMIint; \\
\hline
\end{tabular}

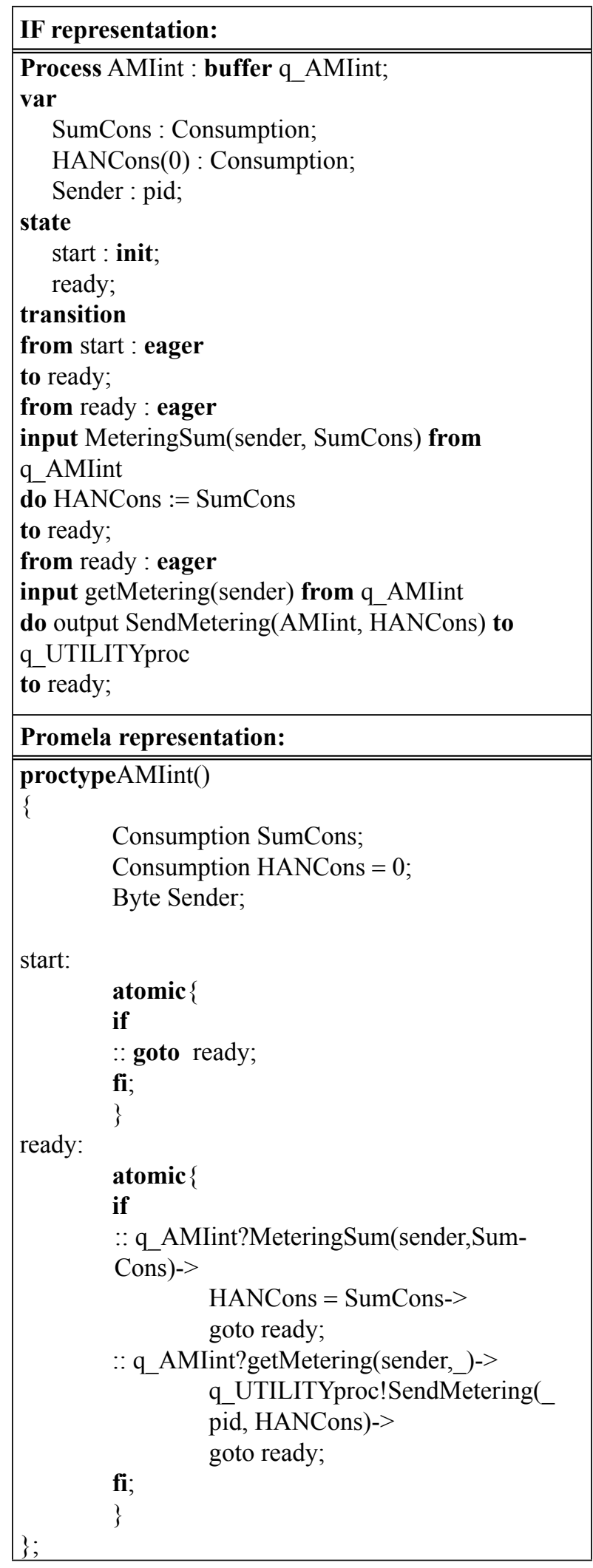

\section{Verification by Model Checking}

The model checking is the most powerful and successful approach to verify requirements and 
correctness of a system hard to build. It brings a lot of benefits to users over other methods such as testing and simulation. In fact, testing and simulation cannot cover all the possible cases, scenarios and behaviors; and generally it is impossible to build the system under consideration to make these tests (e.g. a complex system like Smart Grid). So, these techniques are not exhaustive, and the problem or the failure cases may be among those not tested or simulated scenarios. Contrariwise, the model checking technique allows verifying the whole system automatically. The verification terminates normally or produces a counterexample in case of failure. This counterexample details reasons why the model does not satisfy the specification. The source of errors is detected by studying it. The idea behind the model checking is to ensure that a given model satisfies enough system properties; so that a designer can increase the confidence in the correctness of the model.

Once the DTPromela model was generated, the verification and validation step using DTSPIN (version 4.1.1) was used to check the correctness of the model. DTSPIN was installed under Solaris machine, with 5 GB of memory.

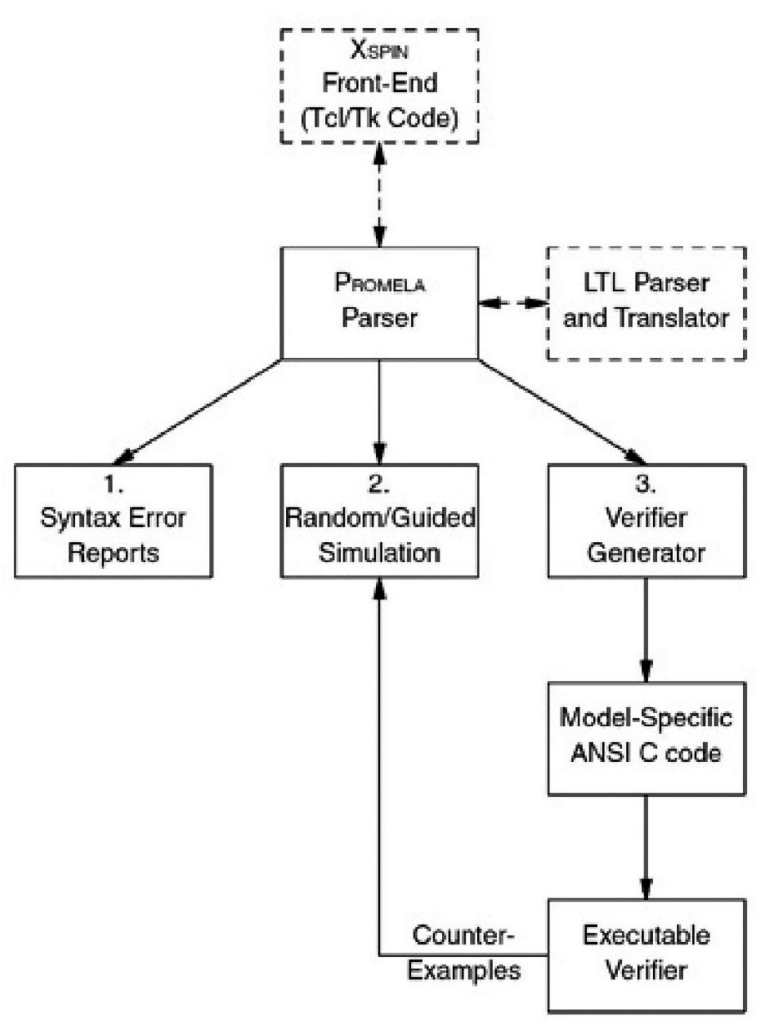

Figure 6. The structure of SPIN [34].
This verification was performed with the help of a graphical interface named XSpin (version 4.1.1) [34]. The XSpin was used, even if it is no longer supported, because it is the only graphical interface compatible with the DTSPIN. The interface was developed in tcl/tk. It is independent from DTSPIN itself, but it executes DTSPIN command in background and provides graphical displays of the message flows, data values, and other options. Figure 6 illustrates the basic structure of SPIN (and DTSPIN as well) [34].

Given a specification written in Promela, SPIN is used, firstly to find and fix syntax errors. After that, interactive or random simulation is performed to gain the basic confidence that the model meets the intended proprieties. Then, an optimized verifier called pan [35] is generated. This verifier is compiled, with possible compile-time choices for the types of reduction algorithms to be used, and executed to detect counterexamples. In case the counterexamples are detected, a trail file is generated. The guided simulation is then used to detect the source of violation. The verification can be also performed with the linear temporal logic (LTL). Correctness claims are generated from a logic formula in order to verify specific properties of the system.

\subsection{General Verification}

This subsection describes "general" verification, which means launching simulations and verifications without fixing specified properties to be checked. As a first step to verify the model, random simulation was launched. The latter was used to debug a model. Some assert statements (e.g. to check that a variable is never taking a negative value) were defined to be checked. It allows also tracking the channels buffer, the value of global and local variables. Other than assert statements, no correctness requirements were checked during simulation runs. All nondeterministic decisions were resolved randomly. The simulation was repeated with different and random values of "Seed" to obtain different types of runs. The interactive simulation was not launched because the system is complex and it consists of a huge number of states. 
As mentioned earlier, after gaining basic confidence that the model has the intended proprieties, verification was performed by generating an optimized verifier. The latter was used to execute three main search modes: exhaustive verification, bitstate approximation, or hash-compact [18]-[34]. Exhaustive verification can deliver the strongest possible verification result; however, the available memory in the machine did not allow completing the run because of the large state space size. Thus, the bitstate was chosen to identify correctness violations if they existed. This technique was used with the default Partial Order Reduction (POR) [36] to reduce the state space sufficiently. In fact, the idea behind the POR is to reduce the size of the state space that needs to be searched. It exploits the commutativity of concurrently executed transitions, which results in the same state when executed in different orders. This method constructs a reduced state graph. The full state graph, which may be too big to fit in memory, is never constructed. The implementation of this reduction method is out of the scope of this paper. However, justification of the reduction method shows that the behaviors that are not present do not add any information [36]. The hash-compact could be used if the verifier still runs out of memory before it can complete the search with bitstate exploration. This is not the case for the model.

By default, XSpin searches for safety properties only (i.e. under certain conditions, something never occurs), however, when changing the tool options, liveness properties can be checked as well (i.e. under certain conditions, something will ultimately occur). The safety properties assert that the system always stays within some allowed region [37]. Thus, the observed behavior always stays within some allowed set of finite behaviors in which nothing "bad" happens. On the other hand, liveness properties are defined as the set of properties the system must satisfy. So, it defines the good things that capture the required functionality of a system [37]. For example, to prove the absence of assertion violations and deadlock states is a safety property; and to prove the absence of non-progress cycles is a liveness property.

This general verification was terminated without detecting any violations. Next subsection describes the verification using the LTL properties.

\subsection{DR Service Properties and LTL Formulas}

After general verification of the model, the next step is verification of desirable properties written in LTL [38]. In LTL, the properties of a run are formalized, unambiguously and concisely, with the help of a small number of special temporal operators [39]-[40]-[41]. Thus, an LTL property is a temporal logic formula that describes a set of infinite sequences for which it is true (e.g., a condition will eventually be true, a condition will be true until another fact becomes true, etc).

This subsection presents some of these properties in relation to the DR Service. They represent requirements captured from the model specification. Nine temporal formulae were defined to help building the desired properties:

- $p 1$ (Store $>=0)$

- $\mathrm{p} 2($ Battery $>=0)$

- $\mathrm{p} 3$ (EVexist $==$ false)

- p4 (PlugEV@ready)

- p5 (SmartPlug@ready)

- p6 (q_DemandResponse?[RTP])

- p7 (DemandResponse@DecisionStat)

- p8 (q_DRint?[Bid])

- $\quad$ p9 (q_DemandResponse?[Bid] || q_UTILITYproc?[AcceptBid])

\section{Property 1: [] p1}

The variable Store refers to the amount of energy generated by the renewable energy sources and stored in the customer house. Several concurrent processes influence this variable either by increasing or decreasing its value. This value must always be positive.

\section{Property 2: [] p2}

The customer can also save energy stored in an electrical vehicle. So, same as the first property, the Battery variable, which represents the electrical vehicle charge, must not be negative.

The first and the second properties are safety properties. They represent unwanted situations (i.e. negative value for variable) that should never occur. Furthermore, the above safety properties are of a particular kind; they are in- 
variants. In fact, invariants are the properties given by a condition and require that this latter holds for all reachable states.

\section{Property 3: [] $>$ p5}

The SmartPlug process waits for the devices to be turned on. After that, it registers them in the data base. The process must not hang at the registration state for a long time because other devices may be connected to the system at this time. This property states that if $\mathrm{p} 5$ (i.e. SmartPlug is at the state ready) happens to be false at any given point in a run, it is always guaranteed to become true again if the run is continued.

\section{Property 4: [] $<$ p4}

This property is same as the previous one, but for an electrical vehicle. It ensures that PlugEV eventually always returns to the state ready.

The third and fourth properties are liveness type. They define the positive behaviors indicating the required functionality of the system.

\section{Property 5: [] (p3 -><> p4)}

The electrical vehicle EV connected to the network may be unplugged by the customer at any time. The connection of EV with the network is represented by a global Boolean EVexist. The latter can be modified by several processes. It must be guaranteed that once the variable value become false, the process PlugEV stops all activities and returns to the ready state.

\section{Property 6: [] (p6 ->> p7)}

In Time-based Programs (RTP) the electricity price changes for different periods. It depends on customers' choice to decrease or change their consumption in response to price changes during a period. When a customer receives an RTP signal, the process DemandResponse chooses the devices to be turned off, if any, and reaches the decision state. The algorithm used in this process, and described in a previous work [3], is composed of many loops. It must be guaranteed that the process can reach the decision state at certain times.

\section{Property 7: [] (p8 ->>> p9)}

In the Incentive-based Programs (Bid) the actions are initiated by the utility or the DR Service Provider. DR signals are sent to every customer participating in one of these pro- grams, in order to motivate him/her to reduce their energy consumption. In exchange for this diminution, customers will benefit from an incentive payment, bill creditor contractual arrangements between them and the electricity suppliers. Generally, DR signals are sent in the peak hours. They may either be voluntary demand reduction requests or mandatory commands. When a customer receives one of these signals, the ESI interface must either refuse the request, if the offer does not meet the user's need, or accept it and send the bid to HEMS to check the possibility of selling energy to utility.

The last three properties are also liveness properties. However, they are of special type called response. When verifying a liveness property with DTSPIN, it is important to switch to weak fairness [42]. This means that every process that is almost always enabled should be executed infinitely often. In other words, it expresses that under certain conditions, something will (or will not) occur infinitely often. DTSPIN accepts only weak fairness (which is sufficient in this work).

It should be noted that XSpin generates a never claim from each property before to be verified. All the seven properties are desired, so, they are negated before generating the never claim. For instance, the last property [] (p8 $->>p$ p) is negated and then converted to the never claim as follows:

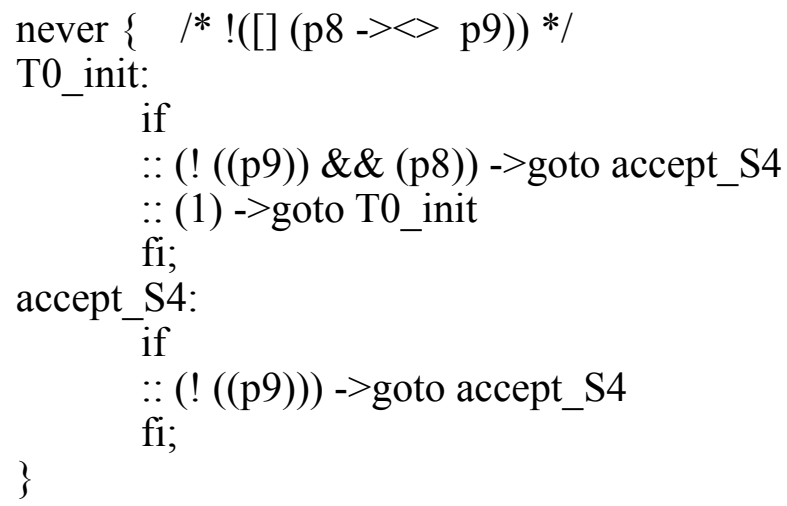

The result of the verification has showed that the model is too complex. In fact, this is clear from Table 3, which shows the number of states searched while verifying the properties. It presents the states stored (i.e. total number of unique global system states stored in the state space), and states matched (i.e. how 
Table 3. Number of states explored by DTSPIN.

\begin{tabular}{|c||c||c|}
\hline Property & States stored & States matched \\
\hline \hline 1 & $4.84004 \mathrm{e}+07$ & $4.39905 \mathrm{e}+08$ \\
\hline 2 & $4.84004 \mathrm{e}+07$ & $4.39905 \mathrm{e}+08$ \\
\hline 3 & $4.82335 \mathrm{e}+07$ & $3.60119 \mathrm{e}+08$ \\
\hline 4 & $4.81368 \mathrm{e}+07$ & $3.53796 \mathrm{e}+08$ \\
\hline 5 & $4.82335 \mathrm{e}+07$ & $3.60119 \mathrm{e}+08$ \\
\hline 6 & $4.83146 \mathrm{e}+07$ & $4.4187 \mathrm{e}+08$ \\
\hline 7 & $4.84947 \mathrm{e}+07$ & $4.06158 \mathrm{e}+08$ \\
\hline
\end{tabular}

many times the search returns to a previously visited state in the search tree).

During the verification, DTSPIN detected unreached states in the processes, as shown in Table 4. For each process, the number of unreached states is indicated between brackets. Generally, in a full state space search, the unreached states indicate a dead code (i.e. transitions are unreachable). However, this is not the case here. In fact, the search depth was set to 10000 , which was imposed by the available memory in the operating system (i.e. 5 GB). If a higher value was used, pan verifier got out of the memory. Despite not detecting any violation, this is a limitation of this verification.

Verification with DTSPIN took several hours to complete, when all available memory was used. This problem is due to dynamic creation of processes. In fact, each time a device is off, it can be turned on by the user. Thus, a new process with new PID is created. In order to fix this, the number of "PowerOFF" signal sent in the system was limited. The same thing was done with the EV, so only one vehicle was plugged at a time. This ensures that the processes will be created and ended only once. It is important to note here that, unlike SDL, Promela distinguishes between the termination of process and the end of its execution. A process terminates (i.e. releases the resources allocated to it) when all younger processes have terminated first. It is impossible to force a process to terminate because it depends on other processes. However, in this verification, the process was forced to end by making it
Table 4. Unreached states.

\begin{tabular}{|l||c|c|c|c|c|c|c|}
\hline Property & 1 & 2 & 3 & 4 & 5 & 6 & 7 \\
\hline _Timers (5) & 1 & 1 & 1 & 1 & 1 & 1 & 1 \\
\hline SmartPlug (94) & 3 & 3 & 4 & 24 & 4 & 4 & 3 \\
\hline Device (37) & 4 & 4 & 9 & 13 & 9 & 5 & 4 \\
\hline DeviceDB (30) & 2 & 2 & 5 & 7 & 5 & 4 & 2 \\
\hline DemandResponse (178) & 19 & 19 & 29 & 32 & 29 & 27 & 34 \\
\hline EnergyUse (59) & 9 & 9 & 9 & 9 & 9 & 9 & 9 \\
\hline AMIint (14) & 1 & 1 & 1 & 1 & 1 & 1 & 1 \\
\hline DRint (79) & 4 & 4 & 3 & 3 & 3 & 4 & 4 \\
\hline Metering (12) & 2 & 2 & 2 & 2 & 2 & 2 & 2 \\
\hline SetAndDisplay (52) & 3 & 3 & 5 & 4 & 5 & 3 & 3 \\
\hline PlugEV (112) & 4 & 4 & 29 & 13 & 29 & 10 & 7 \\
\hline EV (101) & 17 & 17 & 33 & 27 & 33 & 27 & 21 \\
\hline CUSTOMERproc (197) & 1 & 1 & 1 & 1 & 1 & 1 & 1 \\
\hline ENVproc (24) & 1 & 1 & 1 & 1 & 1 & 1 & 1 \\
\hline UTILITYproc (74) & 1 & 1 & 1 & 1 & 1 & 1 & 1 \\
\hline
\end{tabular}

reach the end bracket (i.e. goto LABEL). The process may be not terminating, but it no longer has any interaction with others.

After the modification, verification was run again, with a search depth of 1000000 . This time it finished in six hours (in average) without detecting any violations, but the pan was again out of the memory. Thus, to be sure that the model is free of errors, the stack cycling method [34] is enabled. This method is useful for verifications that require a very large depth limit, which is the case in this work. When the stack cycling is enabled, only a small fraction of the stack is kept in the memory, while the unused portions of the search stack are stored on the disk. With this method, the pan did not run out of memory. When this option was used with the first LTL property, the verification took more than 48 hours, without detecting any violation. The problem of the verification time is due to the process termination as mentioned before. 


\section{Conclusion}

This paper presented a detailed description of a case study in order to validate an SDL HAN model. It proposed a semi-automatic way to generate the Promela model from the HAN SDL model detailed in Section 2. An intermediate format IF was used in order to facilitate this translation. This latter was described in two steps. The first one is manual mapping between SDL and IF. Translation features concerning the structure and behavior and the data type were defined. The "nostable" states were used to decompose each transition into simple actions, in order to reduce combinatorial explosion. However, even with this solution, the manual translation from the SDL model into IF is still a source of potential errors. It is difficult to guarantee that the IF model is free of such errors. The generated IF model was then transformed into DTPromela, using the if $2 \mathrm{pml}$ tool. The latter supplements the model with a definition of Timer as a Promela macro. Once the DTPromela model was prepared, the verification and validation were performed. Desired properties to be checked were defined in LTL, and DTSPIN was used to check the correctness of the model. In the first place, pan got out of memory, even when limiting the number of processes created. However, to solve this problem, an algorithm built in SPIN was used to keep only a small fraction of the search stack in the memory, but the verification took several hours to finish.

Our future work aims to generate the $\mathrm{C}$ code from the HAN SDL specification. To do this, one of the SDL to C compiler from the IBM Rational SDL Suite will be used. The environment functions will be designed in order to connect the SDL system with its environment. The goal of this step is to build either a simulator/emulator core or a plug-in for the HAN network.

\section{References}

[1] S. Zahid et al., "A Synthesis of Communication Architectures and Services of Smart Grid Systems", 3rd Int Conf on Syst of Collaboration (SysCo). IEEE, 2016, pp. 1-6.

http://dx.doi.org/10.1109/SYSCO.2016.7831334
[2] US Dept of Energy, "Communications Requirements of Smart Grid Technologies", Tech. Rep, pp. 1-69, 2010.

http://energy.gov/gc/downloads/communications-requirements-smart-grid-technologies

[3] S. Zahid et al., "SDL Modeling and Validation of Home Area Network in Smart Grid Systems", The Mediterranean Symp on Smart City Applicat. Springer, pp. 28-43, 2017. https://doi.org/10.1007/978-3-319-74500-8_3

[4] B. Vlaovič et al., "Automated Generation of Promela Model from SDL Specification", Comput Standards \& Interfaces, vol. 29, pp. 449-461, 2007.

http://dx.doi.org/10.1016/j.csi.2006.10.001

[5] E. Clarke et al., "Model Checking", MIT Press, 2018.

[6] N. Good et al., "Review and Classification of Barriers and Enablers of Demand Response in the Smart Grid", Renew. Sustain. Energy Rev., vol. 72, pp. 57-72, 2017. https://doi.org/10.1016/j.rser.2017.01.043

[7] N. Paterakis et al., "An Overview of Demand Response: Key-Elements and International Experience", Renewable and Sustainable Energy Reviews, vol. 69, pp. 871-891, 2017. http://dx.doi.org/10.1016/j.rser.2016.11.167

[8] F. Belina and D. Hogrefe, "The CCITT-Specification and Description Language SDL", Comput. Networks ISDN Syst., vol. 16, pp. 311-341, 1989. http://dx.doi.org/10.1016/0169-7552(89)90078-0

[9] M. Fantinato and M. Jino, "Applying Extended Finite State Machines in Software Testing of Interactive Systems", Int Workshop on Design, Specification, and Verification of Interactive Syst. Springer, Berlin, Heidelberg, 2003, pp. 34-45. http://dx.doi.org/10.1007/978-3-540-39929-2_3

[10] P. F. I. Casas et al., "Formalizing Geographical Models Using Specification and Description Language: The Wildfire Example," Proc. of the 2013 Winter Simulation Conf: Simulation: Making Decisions in a Complex World. IEEE Press, 2013, pp. 1961-1972.

http://dx.doi.org/10.1109/WSC.2013.6721575

[11] C. Baier and J.-P. Katoen, "Principles of Model Checking.", MIT press, 2007.

[12] M. Frappier et al., "Comparison of Model Checking Tools for Information Systems", in Int Conf on Formal Eng Methods. Springer, Berlin, Heidelberg, 2010, pp. 581-596. http://dx.doi.org/10.1007/978-3-642-16901-4_38

[13] D. Jackson, "Software Abstractions: Logic Language and Analysis", Cambridge, MA, USA: MIT Press, 2006.

[14] H. Garavel et al., "CADP 2011: A Toolbox for the Construction and Analysis of Distributed Pro- 
cesses", Int. J. Softw. Tools Technol. Transfer, vol. 15, no. 2, pp. 89-107, 2013. http://dx.doi.org/10.1007/s10009-012-0244-z

[15] A.W. Roscoe, "The Theory and Practice of Concurrency", Prentice Hall, 1998.

[16] A. Cimatti et al., "NuSMV 2: An OpenSource Tool for Symbolic Model Checking", in Proc. of the Int Conf on Comput-Aided Verification (CAV 2002), Copenhagen, Denmark, 2002. http://dx.doi.org/10.1007/3-540-45657-0_29

[17] M. Leuschel et al., "ProB: A Model Checker for B" in Int Symp of Formal Methods Europe. Springer, Berlin, Heidelberg, 2003, pp. 855-874. http://dx.doi.org/10.1007/978-3-540-45236-2_46

[18] G. J. Holzmann, "The Model Checker SPIN", IEEE Transactions on Software Eng, vol. 23, no. 5, pp. 279-294, 1997. http://dx.doi.org/10.1109/32.588521

[19] F. Mazzanti and A. Ferrari, "Ten Diverse Formal Models for a CBTC Automatic Train Supervision System," Electron Proc in Theoretical Comput Sci, vol. 268, pp. 104-149, 2018.

http://dx.doi.org/10.4204/EPTCS.268.4

[20] M. H. T. Beek et al., "From EU Projects to a Family of Model Checkers," Software, Services, and Syst Lecture Notes in Comput Sci, pp. 312-328, 2015. https://doi.org/10.1007/978-3-319-15545-6_20

[21] J. F. Groote and M. R. Mousavi, "Modeling and Analysis of Communicating Systems", The MIT Press, 2014. http://dx.doi.org/10.7551/mitpress/9946.001.0001

[22] K. Jensen and L. Kristensen, "Coloured Petri Nets: Modelling and Validation of Concurrent Systems", Springer Science \& Business Media, 2009.

http://dx.doi.org/10.1007/b95112

[23] FDR4 - The CSP Refinement Checker. http://www.cs.ox.ac.uk/projects/fdr/

[24] L. Lamport, "Specifying Systems: The TLA+ Language and Tools for Hardware and Software Engineers", MA, Reading: Addison-Wesley, 2002.

[25] K. Larsen et al., "UPPAAL in a Nutshell", Int Journal on Software Tools for Technology Transfer (STTT), vol. 1, no 1, pp. 134-152, 1997. http://dx.doi.org/10.1007/s100090050010

[26] Blaskovic et al., "Model Checking of Concurrent System with SDL/sup --/ Specification," IEEE 10th Mediterranean Electrotechnical Conf. Inform Technology and Electrotechnology for the Mediterranean Countries. Proc. of the MeleCon 2000 (Cat. No.00CH37099), 2000, vol. 1, pp. 77-80.

http://dx.doi.org/10.1109/MELCON.2000.880372
[27] D. Bošnački et al., "Model Checking SDL with Spin", Proc. of the TACAS'2000, 2000, vol. 1785, pp. 363-377.

https://doi.org/10.1007/3-540-46419-0_25

[28] A. Hessel, "Timing Analysis of sdl Subset in Uppaal," Master Thesis, Uppsala University, 2002.

[29] M. Bozga et al., "IF: An Intermediate Representation for SDL and its Applications", in SDL Forum, pp. 423-40, 1999.

http://dx.doi.org/10.1016/B978-044450228-5/50028-X

[30] A. Prigent et al., "Extending the Translation from SDL to Promela", in Proc. of the 9th Int SPIN Workshop on Model Checking of Software, 2002, pp. 79-94. http://dx.doi.org/10.1007/3-540-46017-9_8

[31] B. Vlaovič et al.,"Applying Automated Model Extraction for Simulation and Verification of Real-Life SDL Specification with Spin", IEEE Access, vol. 5, pp. 5046-5058, 2017. http://dx.doi.org/10.1109/ACCESS.2017.2685238

[32] A. Vreže et al., "Sdl2pml - Tool for Automated Generation of Promela Model From SDL Specification", Comput Standards \& Interfaces, vol. 31, no. 4, pp. 779-786, 2009.

https://doi.org/10.1016/j.csi.2008.09.005

[33] S. Graf et al., "Model Checking of UML Models via a Mapping to Communicating Extended Timed Automata" in S. Graf and L. Mounier (Eds.), Proc. of the SPIN'04 Workshop, Barcelona, Spain, vol. 2989 of LNCS, Springer, 2004.

[34] G. J. Holzmann, "The SPIN Model Checker: Primer and Reference Manual"., Addison-Wesley, 2003.

[35] G. J. Holzmann, "PAN: A Protocol Specification Analyzer", Technical Rep TM81-11271-5, AT\&T Bell Laboratories, 1981.

[36] D. Baeld et al., "Partial Order Reduction for Security Protocols", in Proc. of the 26th Int Conf on Concurrency Theory (CONCUR'15) ser. LIPIcs, 2015, vol. 42, pp. 497-510. https://dx.doi.org/10.4230/LIPIcs.CONCUR.2015.497

[37] I. Konnov et al., "A Short Counterexample Property for Safety and Liveness Verification of Fault-Tolerant Distributed Algorithms" in Proc. of the 44th ACM SIGPLAN Symp on Principles of Programming Languages - POPL 2017, 2017. https://dx.doi.org/10.1145/3009837.3009860

[38] E. M. Clarke and E. A. Emerson, "Design and Synthesis of Synchronization Skeletons for Branching Time Temporal Logic", in Proc. of the Logic of Programs: Workshop, 1981. https://doi.org/10.1007/BFb0025774

[39] M. Dwyer et al., "Property Specification Patterns for Finite-State Verification", Proc. of the 2nd 
Workshop on Formal Methods in Software Practice, 1998, pp. 7-15. http://dx.doi.org/10.1145/298595.298598

[40] A. Pnueli, "The Temporal Logic of Programs", Proc. of the 18th IEEE Symp. Foundations of Comput Sci, 1977, pp. 46-57. http://dx.doi.org/10.1109/SFCS.1977.32

[41] E. M. Clarke et al., "Another Look at LTL Model Checking" in Int Conf on Comput Aided Verification, Springer, Berlin, Heidelberg, 1994, pp. 415-427.

http://dx.doi.org/10.1007/3-540-58179-0_72

[42] V. Germanos et al., "Diagnosability under Weak Fairness", 14th Int. Conf. on Application of Concurrency to Sys Design, 2014, pp. 132-141. http://dx.doi.org/10.1109/ACSD.2014.9
Contact addresses:

Soufiane Zahid Institut National des Postes et Télécommunication

Rabat

Morocco

e-mail: zahidsoufiane@gmail.com

Abdeslam En-Nouaary
Institut National des Postes et Télécommunication
Rabat
Morocco
e-mail: abdeslam@inpt.ac.ma

Slimane Bah

Ecole Mohammadia d'Ingénieurs University Mohammed V

Rabat

Morocco

e-mail: slimane.bah@emi.ac.ma
Received: October 2018

Revised: October 2019 Accepted: November 2019
Soufiane ZahiD received his Diploma (engineer degree) in Telecommunication, option Networks and Computer Systems, from the Institut National des Postes et Télécommunication (INPT), Rabat, Morocco, in 2012. He is currently a PhD student in the doctoral center of INPT. His current research topics are smart grid networks, formal methods and conceptual modeling.

ABdeslam En-NouaARY received his engineer degree in computer engineering, option Data Communication and Computer Networks, from the École Nationale Supérieure d'Informatique et d'Analyse des Systèmes (ENSIAS), Rabat, Morocco, in 1996, and the MSc and PhD degrees in computer science from the University of Montreal, in 1998 and 2001, respectively. Dr. En-Nouaary is currently an Associate Professor at INPT (Institut National des Postes et Télécommunications), Rabat, Morocco. Before joining INPT in 2008, Dr. En-Nouaary had been an Associate Professor at the Electrical and Computer Engineering Department of Concordia University, Montreal, Canada, from 2001 to 2008. His main research interests are modeling and validation of distributed, real-time, and embedded systems.

Slimane Bah holds a PhD in computer networks from Concordia University, Montreal, Canada. During his PhD research he was an intern at Ericsson Canada. Dr. Bah also holds an MSc in computer networks from the University of Montreal, Canada, and an engineering degree in computer science from the École Nationale Supérieure d'Informatique et d'Analyse des Systèmes (ENSIAS), Morocco. Currently, Dr. Bah is an Associate Professor at the Computer Science Department of Mohammadia Engineering School (Ecole Mohammadia d'Ingenieurs), University Mohammed V in Rabat, Morocco. He has also worked as an Adjunct Professor at the University of Moncton, Canada. His research interests include end-user services, self-organizing and challenging networks, service and protocol engineering. 